\title{
NUTRIÇÃO MINERAL E PRODUÇÃO DE ABACAXIZEIRO 'PÉROLA', EM FUNÇÃO DAS RELAÇÕES K/N NA ADUBAÇÃO'
}

\author{
ALESSANDRA ALVES RODRIGUES ${ }^{2}$, REJANE MARIA NUNES MENDONÇA ${ }^{3}$ \\ ALEXANDRE PAIVA DA SILVA ${ }^{4}$, SILVANDA DE MELO SILVA ${ }^{5}$
}

RESUMO-A adubação do abacaxizeiro deve contemplar a reposição das quantidades de $\mathrm{N}$ e $\mathrm{K}$ absorvidas pela planta e exportadas pelos frutos e mudas, e o estabelecimento de relações adequadas entre as doses destes nutrientes. Este trabalho objetivou avaliar o efeito de diferentes relações $\mathrm{K} / \mathrm{N}$ na adubação sobre a nutrição mineral e a produção de abacaxizeiro 'Pérola', em solos de Tabuleiros Costeiros da Paraíba. O experimento foi conduzido em delineamento experimental em blocos casualizados com 13 tratamentos e três repetições. Os tratamentos resultaram da combinação de quatro relações $\mathrm{K} / \mathrm{N}(0,85: 1 ; 1: 1 ; 2: 1$ e $3: 1)$, duas doses de $\mathrm{N}$ (7,2 e 10,8 g planta $^{-1}$ ), quatro tratamentos adicionais (relação $\mathrm{K} / \mathrm{N}$ de 2:1 na maior dose de $\mathrm{N}$, variando-se a fonte, o parcelamento, a época e a forma de aplicação das doses de $\mathrm{N}$ e K) e uma testemunha absoluta (sem adubação). $\mathrm{O}$ aumento das relações $\mathrm{K} / \mathrm{N}$ eleva o peso da folha ' $\mathrm{D}$ ', os teores de $\mathrm{K}$ e os valores das relações $\mathrm{K} / \mathrm{N}$ e $\mathrm{K} / \mathrm{Mg}$; reduz os teores de $\mathrm{N}$ e não afeta os teores de $\mathrm{P}$, Ca e a relação $\mathrm{K} / \mathrm{Ca}$. O aumento das relações $\mathrm{K} / \mathrm{N}$ não influencia no peso médio, na produtividade e no percentual de frutos das classes I, II e III. A utilização de sulfato de $\mathrm{K}$, o parcelamento das doses de $\mathrm{N}$ e $\mathrm{K}$, na forma de $\mathrm{KCl}$, em cinco aplicações, e a aplicação de metade das doses de $\mathrm{N}$ e $\mathrm{K}$ via foliar na relação 2:1 aumentam o peso da folha ' $\mathrm{D}$ ', o teor foliar de $\mathrm{N}$ e os valores de peso médio, produtividade e percentual de frutos da classe II.

Termos de indexação: Ananas comosus comosus, nitrogênio, potássio.

\section{MINERAL NUTRITION AND YIELD OF PINEAPPLE PLANT CV. PÉROLA AS A FUNCTION OF THE K/N RELATIONSHIPS IN FERTILIZATION}

\begin{abstract}
The pineapple fertilization should include the replacement of the amounts of $\mathrm{N}$ and $\mathrm{K}$ absorbed by the plant and exported by the fruits and slips, and appropriate relations between doses of these nutrients. This study evaluated the effect of different $\mathrm{K} / \mathrm{N}$ on mineral nutrition and yield of pineapple 'Perola' in Coastal Tablelands soils of Paraiba. The experiment was conducted in a randomized complete block design with 13 treatments and three replications. The treatments consisted of combinations of four $\mathrm{K}$ / $\mathrm{N}\left(0.85: 1,1: 1,2: 1\right.$ and 3:1), two $\mathrm{N}$ levels (7.2 and $\left.10.8 \mathrm{~g} \mathrm{plant}^{-1}\right)$, four additional treatments $(\mathrm{K} / \mathrm{N}$ of $2: 1$ at the highest dose of $\mathrm{N}$, varying the source, the subdivision, date and manner of application of both $\mathrm{N}$ and $\mathrm{K}$ ) and a control (without fertilization). The increase in $\mathrm{K} / \mathrm{N}$ increase the weight of the ' $\mathrm{D}$ ' leaf, the contents of $\mathrm{K}$ and the values for $\mathrm{K} / \mathrm{N}$ and $\mathrm{K} / \mathrm{Mg}$; decreased the contents of $\mathrm{N}$, but did not affect the content of $\mathrm{P}$, $\mathrm{Ca}$ and the relationship $\mathrm{K} / \mathrm{Ca}$. The increase in $\mathrm{K} / \mathrm{N}$ did not affect weight, productivity and the percentage of fruit class I, II and III. The use of $\mathrm{K}$ sulfate, split doses of $\mathrm{N}$ and $\mathrm{K}$ as $\mathrm{KCl}$, in five applications and the application of half doses of foliar $\mathrm{N}$ and $\mathrm{K}$ in a ratio of 2:1 increase the weight of the ' $\mathrm{D}$ ' leaf, the contents of $\mathrm{N}$ and values of average weight, productivity and the percentage of fruit class II.
\end{abstract}

Index terms: Ananas comosus comosus, nitrogen, potassium.

1(Trabalho 220-12). Recebido em: 05-07-2012. Aceito para publicação: 28-03-2013.

${ }^{2}$ Agrônoma, Doutora em Agronomia, UFPB, Centro de Ciências Agrárias, Campus II, 58.397-000 / Areia-PB. E-mail:alessaagro@ gmail.com

${ }^{3}$ Prof. da Universidade Federal da Paraíba, Campus II, Areia-PB, E-mail: rejane@cca.ufpb.br, silvasil@cca.ufpb.br

${ }^{4}$ Prof. da Universidade Federal de Campina Grande, CCTA/UACTA, Pombal-PB. E-mail: paivadasilva@gmail.com 


\section{INTRODUÇÃO}

A abacaxicultura representa o segmento frutícola de maior importância no Estado da Paraíba, atualmente o segundo maior produtor nacional, com uma produção de 274 milhões de frutos, numa área colhida de 60 mil ha (IBGE, 2012). O abacaxizeiro é cultivado nas microrregiões litorâneas, em áreas de Tabuleiros Costeiros, nas quais predominam solos de textura arenosa, ácidos e de baixa fertilidade natural (SOUZA et al., 2007; RODRIGUES et al., 2010). A produção é feita basicamente por pequenos produtores (áreas $<5 \mathrm{ha}$ ), sem irrigação, utilizando-se da cultivar Pérola (SOUZA et al., 2007; RODRIGUES et al., 2010).

Potássio e nitrogênio são os nutrientes requeridos em maiores quantidades pelo abacaxizeiro e desempenham isolados e/ou associados funções importantes nos aspectos nutricionais da cultura, os quais afetam diretamente o crescimento vegetativo, a produção e a qualidade dos frutos (RAZZAQUE; HANAFFI, 2001; VELOSO et al., 2001; TEIXEIRA et al., 2002; MALÉZIEUX; BARTHOLOMEW, 2003; SPIRONELLO et al., 2004; SOARES et al., 2005). Contudo, tais efeitos são influenciados, dentre outros fatores, pelas fontes, doses, forma e épocas de aplicação dos fertilizantes utilizados na adubação (CHOAIRY et al., 1990; RAZZAQUE; HANAFFI, 2001; TEIXEIRA et al., 2002; SPIRONELLO et al., 2004; SOARES et al., 2005; TEIXEIRA et al., $2011 \mathrm{ab})$.

A adubação do abacaxizeiro deve contemplar, além da reposição de quantidades de $\mathrm{N}$ e $\mathrm{K}$ absorvidas pela planta e exportadas pelos frutos e mudas, o estabelecimento de relações adequadas entre as doses desses nutrientes (OWUSU-BENNOAH et al., 1997; SOUZA, 2000; MALÉZIEUX; BARTHOLOMEW, 2003). Tal procedimento, nem sempre considerado nas recomendações de adubação, consiste numa estratégia importante para assegurar o equilíbrio nutricional da cultura, visando a maximizar as produtividades física, econômica e de qualidade (MALÉZIEUX; BARTHOLOMEW, 2003; SILVA et al., 2009).

Apesar de comuns para outras culturas, são bastante escassas na literatura as informações sobre a resposta do abacaxizeiro às variações das relações $\mathrm{K} / \mathrm{N}$ entre as doses (SOUZA, 2000; SILVA et al., 2009). Em solos da Savana Africana, Owusu-Bennoah et al. (1997) avaliaram os efeitos de três relações $\mathrm{K} / \mathrm{N}(1,5: 1 ; 2: 1$ e $2,5: 1)$, utilizando seis doses de $\mathrm{K}\left(336 ; 448 ; 504 ; 560 ; 672\right.$ e $840 \mathrm{~kg} \mathrm{ha}^{-1}$ de $\mathrm{K}_{2} \mathrm{O}$, cloreto de potássio) e duas doses de N (224 e 336 $\mathrm{kg} \mathrm{ha}^{-1}$, sulfato de amônio) sobre a nutrição mineral e a produção do abacaxizeiro 'Smooth Cayenne'. Verificaram que, com exceção dos teores foliares de $\mathrm{N}\left(<11 \mathrm{~g} \mathrm{~kg}^{-1}\right)$ e da relação $\mathrm{K} / \mathrm{N}$ na folha ' $\mathrm{D}$ ' $(\mathrm{K} / \mathrm{N}$ foliar $<3,0)$, os teores dos demais nutrientes $(\mathrm{P}, \mathrm{K}$, $\mathrm{Ca}$ e $\mathrm{Mg}$ ) foram considerados adequados. Observaram ainda que o aumento da relação $\mathrm{K} / \mathrm{N}$ elevou o peso médio dos frutos na menor dose de $\mathrm{N}$, tendo a relação 2,5:1 (560 kg ha-1 de $\mathrm{K}_{2} \mathrm{O}$ e $224 \mathrm{~kg} \mathrm{ha}^{-1}$ de $\mathrm{N})$ se destacado em relação às demais.

No Brasil, informações dessa natureza ainda são incipientes, e as recomendações são, em sua maioria, empíricas, generalistas e com pouca experimentação. Conforme Sousa (2000), devido aos efeitos antagônicos entre $\mathrm{N}$ e K, a opção por determinada relação deve estar baseada no destino da produção. Segundo o autor, para mercados externos, internos distantes e para indústria, devem-se adotar relações mais amplas (entre 1,5 e 2,5), visando a ajustar a relação sólidos solúveis / acidez titulável e conferir maior resistência ao transporte. Entretanto, para mercados menos exigentes e/ou próximos da área produtora, a relação pode ser mais estreita $(\mathrm{K} / \mathrm{N} \leq 1,0)$.

Este trabalho teve por objetivo avaliar o efeito de diferentes relações $\mathrm{K} / \mathrm{N}$, estabelecidas entre as doses destes nutrientes na adubação, sobre a nutrição mineral e a produção de abacaxizeiro 'Pérola', nas condições edafoclimáticas de Tabuleiros Costeiros do Estado da Paraíba.

\section{MATERIAL E MÉTODOS}

O experimento foi conduzido durante o período de março de 2006 a setembro de 2007, em condições de sequeiro, na Propriedade Sertaneja, localizada no município de Santa Rita (7²14'20" S e 34⒌'28" W), região de Tabuleiros Costeiros do Estado da Paraíba. O clima predominante, pela classificação de Köppen, é o As', quente e úmido, com temperaturas médias entre 22 e $26{ }^{\circ} \mathrm{C}$ e precipitação pluviométrica anual de $1.500 \mathrm{~mm}$, concentrada durante os meses de maio a setembro (BRASIL, 1972).

O solo da área experimental está classificado como Espodossolo Humilúvico (EMBRAPA, 2006) e apresentava, na camada de 0-0,20 m, antes da aplicação dos tratamentos, os seguintes atributos físicos (EMBRAPA, 1997) e químicos (TEDESCO et al., 1995): areia $=904,0 \mathrm{~g} \mathrm{~kg}^{-1}$; silte $=72,0 \mathrm{~g} \mathrm{~kg}^{-1}$; argila $=24,0 \mathrm{~g} \mathrm{~kg}^{-1} ;$ densidade do solo $=1,38 \mathrm{~g} \mathrm{~cm}^{-3} ; \mathrm{pH}$ $=4,5 ;$ matéria orgânica $=14,3 \mathrm{~g} \mathrm{dm}^{-3} ;$ P Melhich-1

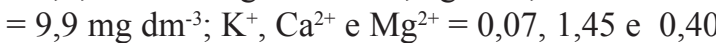
$\mathrm{cmol}_{\mathrm{c}} \mathrm{dm}^{-3}$, respectivamente; $\mathrm{Al}^{3+}=1,0 \mathrm{cmol}_{\mathrm{c}} \mathrm{dm}^{-3}$; $\mathrm{H}+\mathrm{Al}=7,6 \mathrm{cmol}_{\mathrm{c}} \mathrm{dm}^{-3} ; \mathrm{SB}=1,92 \mathrm{cmol}_{\mathrm{c}} \mathrm{dm}^{-3} ; \mathrm{CTC}$ $=9,52 \mathrm{cmol}_{\mathrm{c}} \mathrm{dm}^{-3}, \mathrm{e} \mathrm{V}=20,2 \%$.

$\mathrm{O}$ experimento foi conduzido em delinea- 
mento experimental em blocos casualizados, com 13 tratamentos e três repetições, totalizando 39 unidades experimentais. Os tratamentos resultaram da combinação de quatro relações $\mathrm{K} / \mathrm{N}(0,85: 1 ; 1: 1$; 2:1 e $3: 1)$, duas doses de $\mathrm{N}\left(7,2\right.$ e 10,8 g planta $\left.^{-1}\right)$, quatro tratamentos adicionais (relação $\mathrm{K} / \mathrm{N}$ de $2: 1$ na maior dose de $\mathrm{N}$ para estudar o efeito de fontes, parcelamento, época e forma de aplicação das doses) e uma testemunha absoluta (sem adubação) (Tabela 1). A unidade experimental constou de dez linhas de dez plantas (100 plantas), sendo consideradas como área útil as oito plantas das oito linhas centrais (64 plantas).

As doses de $\mathrm{N}$ e de $\mathrm{K}$ dos tratamentos que avaliaram as relações K:N (tratamentos de um a oito) e dos tratamentos adicionais 09,11 e 12 foram aplicadas via solo, em três aplicações; no entanto, nos tratamentos de um a oito e no tratamento adicional nove (efeito da fonte de K), as doses foram aplicados via solo e igualmente distribuídas aos $60 ; 180 \mathrm{e}$ 270 dias após o plantio. No tratamento adicional 11 (efeito da época de aplicação), as doses foram aplicadas via solo aos 60;180 e 360 dias após o plantio, enquanto no tratamento adicional 12 (efeito da forma de aplicação), as doses foram aplicadas aos 60;180 e 270 dias após o plantio, sendo $50 \%$ via solo e 50 $\%$ via foliar. No tratamento adicional 10 (efeito do parcelamento), as doses foram aplicadas via solo aos 60; 180; 210; 240 e 270 dias após o plantio (Tabela 1). O P foi aplicado em dose única aos 60 dias após o plantio.

Foram utilizadas as seguintes fontes de nutrientes: $\mathrm{N}$ - ureia; $\mathrm{P}_{2} \mathrm{O}_{5}$ - superfosfato triplo e $\mathrm{K}_{2} \mathrm{O}$ - cloreto de potássio e sulfato de potássio (no tratamento 9). As plantas de todos os tratamentos, inclusive as da testemunha, receberam no plantio $500 \mathrm{~g} \mathrm{cova}^{-1}$ de esterco bovino (base matéria seca) e $4 \mathrm{~kg} \mathrm{ha}^{-1} \mathrm{de} \mathrm{B}$, aplicados via foliar, aos 10 meses após o plantio, na forma de bórax. Receberam ainda aplicações foliares de $\mathrm{Fe}, \mathrm{Cu}$ e $\mathrm{Zn}\left(10 \mathrm{~kg} \mathrm{ha}^{-1}\right.$ de sulfato ferroso; $2 \mathrm{~kg} \mathrm{ha}^{-1}$ de sulfato de cobre e $2 \mathrm{~kg}$ ha $^{-1}$ de sulfato de zinco) a partir do quarto mês, em intervalos de dois meses, até o décimo mês após o plantio (OLIVEIRA et al., 2002).

O plantio foi realizado em março de 2006, após preparo do solo, sendo utilizadas mudas do tipo 'filhote' da cultivar Pérola, selecionadas quanto aos aspectos sanitários e padronizadas quanto ao peso $(400 \mathrm{~g})$ e tamanho $(40 \mathrm{~cm})$. As mudas foram tratadas por imersão com Parathion metílico a $0,1 \%$ do princípio ativo (OLIVEIRA et al., 2002) e plantadas no sistema de fileiras simples, no espaçamento de $0,80 \times 0,30 \mathrm{~m}$, perfazendo uma densidade de 41.667 plantas $\mathrm{ha}^{-1}$.
Durante a condução do experimento, as ervas invasoras foram controladas via aplicação do herbicida Diuron, em pós-emergência, e doze capinas manuais a partir do quarto mês após o plantio. Para o controle da cochonilha (Dysmicoccus brevipes), foi aplicado Parathion metílico a 0,1\%, aos 3;7 e 10 meses após o plantio. Para o controle da fusariose, realizaram-se pulverizações semanais com Benomil $\left(60 \mathrm{~g} \mathrm{~L}^{-1}\right)$, dos 45 dias após a indução floral até o fechamento das flores. A indução floral foi feita aos 11 meses após o plantio, mediante aplicação de 50 $\mathrm{mL}_{\text {planta }}{ }^{-1}$ de solução de carbureto de cálcio a $1 \%$, no centro da roseta foliar (OLIVEIRA et al., 2002).

Para avaliar o estado nutricional das plantas, foram coletadas sete folhas ' $\mathrm{D}$ ', em cada unidade experimental, na época de indução floral (11 meses após o plantio). Nas referidas folhas, determinaram-se os valores de massa fresca e retiraram-se subamostras (20 cm centrais) da porção clorofilada para a determinação dos teores de $\mathrm{N}, \mathrm{P}, \mathrm{K}, \mathrm{Ca}$ e $\mathrm{Mg}$ após secagem em estufa a $65^{\circ} \mathrm{C}$ (TEDESCO et al., 1995). Com base nos teores foliares, foram estimados os valores das relações $\mathrm{K} / \mathrm{N}, \mathrm{K} / \mathrm{Ca}$ e $\mathrm{K} / \mathrm{Mg}$.

A colheita foi realizada aos 17 meses após o plantio, quando os frutos atingiram o estádio de maturação 'pintado' (OLIVEIRA et al., 2002). Foram coletados 15 frutos na área útil das unidades experimentais, os quais foram pesados e categorizados nas classes comerciais estabelecidas para a cultivar Pérola: classe I: 0,8-1,2 kg; classe II: 1,21-1,50 kg; classe III: $1,51-1,80 \mathrm{~kg}$; classe IV: > 1,80 kg (CEAGESP, 2003). A produção total foi estimada multiplicandose o percentual de frutos de cada classe comercial pelo respectivo peso médio da classe, considerando uma densidade na colheita de 30.000 plantas $_{\text {ha }}{ }^{-1}$.

Os dados obtidos foram submetidos à análise de variância, e as médias, comparadas pelo teste de Scott-Knott, ao nível de $5 \%$ de probabilidade.

\section{RESULTADOS E DISCUSSÃO}

\section{Peso e teores de nutrientes na folha ' $D$ '}

Os tratamentos influenciaram significativamente sobre os valores de massa fresca da folha ' $D$ ' $(\mathrm{p}<0,01)$ e os teores foliares de $\mathrm{N}$ e K $(\mathrm{p}<0,01)$, sem, no entanto, influenciarem sobre os teores de $\mathrm{P}$, Ca e $\mathrm{Mg}(\mathrm{p}>0,05)$ (Tabela 2).

$\mathrm{O}$ aumento das relações $\mathrm{K}: \mathrm{N}$ elevou a massa fresca da folha ' $\mathrm{D}$ ' nas duas doses de $\mathrm{N}$, sendo o maior valor $(82,8 \mathrm{~g})$ registrado na relação $3: 1$, na menor dose de N (Tabela 2); contudo, este valor não diferiu dos obtidos nas relações 1:1 e 3:1 na maior e da relação 2:1 na menor dose de $\mathrm{N}$, bem como dos tratamentos adicionais; os menores valores foram 
observados nas relações $0,85: 1$ (63,9 g) e 1:1 (65,5 $\mathrm{g}$ ), na menor dose de $\mathrm{N}$ e na testemunha absoluta $(55,9 \mathrm{~g})$ (Tabela 2).

Aumentos nos valores de massa fresca da folha ' $D$ ' com a elevação das doses de $\mathrm{K}$ foram também reportados por Teixeira et al. (2011a) e podem ser explicados pelo papel do K nos processos bioquímicos e fisiológicos do abacaxizeiro, incluindo ativação enzimática, transporte através de membranas, neutralização aniônica e, sobretudo, potencial osmótico da planta. O efeito positivo do $\mathrm{N}$ deve-se ao fato de este nutriente ser constituinte de aminoácidos e proteínas, além de ser indispensável para a utilização de carboidratos no interior da planta, o que contribui para aumentar o número e o peso das folhas (MARSCHNER, 1997).

Considerando a correlação positiva entre massa fresca da folha ' $D$ ' na indução floral e peso do fruto, e que abacaxizeiros 'Pérola' com massa fresca da folha ' $\mathrm{D}$ ' em torno de $80 \mathrm{~g}$ são capazes de produzir frutos com peso superior a $1,2 \mathrm{~kg}$, caso não sejam prejudicados por estresses durante a formação dos frutos (REINHARDT; CUNHA, 2000), inferese, que com exceção das maiores relações $\mathrm{K}: \mathrm{N}$, independentemente da dose de $\mathrm{N}$, e dos tratamentos adicionais, os demais tratamentos exibiram valores inferiores aos mencionados (Tabela 2). Nas mesmas condições edafoclimáticas, Rodrigues et al. (2010) obtiveram peso médio de frutos da cultivar 'Pérola' de $1,57 \mathrm{~kg}$, com valores de peso da folha ' $\mathrm{D}$ ' aos 12 meses após o plantio de $117 \mathrm{~g}$, utilizando doses de $513 \mathrm{~kg} \mathrm{ha}^{-1}$ de N e K.

De forma geral, o aumento das relações K:N, independentemente da dose de $\mathrm{N}$, reduziu significativamente o teores foliares de N (Tabela 2), sem diferirem, entretanto, daqueles obtidos com a aplicação de uma das doses após a indução floral e da testemunha absoluta (Tabela 2). Tendência semelhante foi observada por diversos autores (OWUSU-BENNOAH et al., 1997; TEIXEIRA et al., 2002; SPIRONELLO et al., 2004) e pode ser explicada pelo antagonismo entre $\mathrm{Ne} \mathrm{K}$, pois a distribuição de $\mathrm{N}$ como compostos solúveis e proteínas nas folhas é comprometida pelo aumento dos teores de K (MARSCHNER, 1997).

A elevação das relações $\mathrm{K}: \mathrm{N}$ aumentou significativamente os teores foliares de $\mathrm{K}$, independentemente da dose de N (Tabela 2); contudo, os teores não diferiram dos obtidos nos tratamentos adicionais, tendo a testemunha absoluta apresentado o menor teor de $\mathrm{K}\left(18,8 \mathrm{~g} \mathrm{~kg}^{-1}\right)$. Estes resultados concordam com os obtidos em diversos trabalhos (OWUSU-BENNOAH et al., 1997; TEIXEIRA et al., 2002; SPIRONELLO et al., 2004; TEIXEIRA et al., $2011 \mathrm{ab}$ ) e corroboram as afirmações de Soares et al. (2005) sobre a relação entre teores foliares e disponibilidade de $\mathrm{K}$ no solo ou proveniente da adição de fertilizantes.

Considerando as faixas de teores adequadas para o abacaxizeiro na indução floral (MALÉZIEUX; BARTHOLOMEW, 2003), verifica-se que, com exceção dos teores de $\mathrm{P}$ e K nas maiores relações K:N, nas duas doses de $\mathrm{N}$, e nos tratamentos adicionais, os teores dos demais nutrientes, em todos os tratamentos, encontravam-se abaixo das faixas consideradas adequadas (Tabela 2). Apesar disso, as plantas não apresentaram sintomas de deficiências nutricionais durante o experimento.

Além dos efeitos dos tratamentos (Tabela 2), os baixos teores de $\mathrm{N}$ estão possivelmente relacionados com o aumento da translocação de reservas nutricionais para os frutos (MALÉZIEUX; BARTHOLOMEW, 2003). Por outro lado, os baixos teores de $\mathrm{Ca}$ e $\mathrm{Mg}$ devem-se aos baixos teores destes nutrientes no solo e da ausência de calagem. O antagonismo entre $\mathrm{K}$ e os cátions $\mathrm{Ca}$ e $\mathrm{Mg}$ é bastante conhecido, havendo, em geral, tendência de diminuição dos teores foliares destes nutrientes com a elevação das doses de K (TEIXEIRA et al., 2002), sobretudo em solos com baixos teores de $\mathrm{Ca}$ e $\mathrm{Mg}$ e com aplicações de doses elevadas de $\mathrm{K}$ no solo (MEURER, 2007).

\section{Relações K/N, K/Ca e K/Mg}

Os tratamentos influenciaram sobre os valores das relações $\mathrm{K} / \mathrm{N}(\mathrm{p}<0,01)$ e $\mathrm{K} / \mathrm{Mg}(\mathrm{p}<0,05)$ na folha ' $\mathrm{D}$ ', mas não influenciaram sobre os valores da relação $\mathrm{K} / \mathrm{Ca}(\mathrm{p}>0,05)$ (Tabela 3$)$.

$\mathrm{O}$ aumento das relações $\mathrm{K} / \mathrm{N}$ na maior dose de $\mathrm{N}$ elevou os valores da relação $\mathrm{K} / \mathrm{N}$, os quais não diferiram daqueles do tratamento em que se aplicou uma dose de $\mathrm{N}$ e K após a indução floral (Tabela 3 ). Tais resultados devem-se à elevação dos teores de $\mathrm{K}$ e da diminuição dos teores de $\mathrm{N}$ (Tabela 2), em função do efeito antagônico entre $\mathrm{N}$ e K, o qual teria diminuído os teores de N (MARSCHNER, 1997). Por outro lado, a aplicação de uma dose de N e K após a indução floral deve ter provocado baixa assimilação do N (LACOEUILHE, 1978), resultando em baixos teores de $\mathrm{N}$ e aumento da relação $\mathrm{K} / \mathrm{N}$.

$\mathrm{Na}$ literatura, há relatos de que a relação K/N na folha ' $D$ ', na indução floral, deve ser pelo menos igual a 3,0 (MALÉZIEUX; BARTHOLOMEW, 2003), valor acima dos encontrados neste trabalho. Contudo, Spironello et al. (2004) e Owusu-Bennoah et al. (1997) obtiveram maximização da produção de abacaxizeiro 'Smooth Cayenne', sem comprometimento da qualidade, com relações iguais a 2,4 e 2,7, respectivamente, valores condizentes com os obtidos no presente trabalho (Tabela 3 ). 
A elevação das relações K/N, nas duas doses de $\mathrm{N}$, aumentou os valores da relação $\mathrm{K} / \mathrm{Mg}$ na folha ' $\mathrm{D}$ ', sem que tais valores diferissem daqueles obtidos nos tratamentos adicionais (Tabela 3); os menores valores foram registrados no tratamento-testemunha $(7,6)$ e na relação $\mathrm{K} / \mathrm{N}$ de $0,85: 1$ na menor $(8,4)$ e na maior dose $(9,7)$ de $\mathrm{N}$ (Tabela 3 ).

Diversos autores também encontraram aumentos nos valores da relação $\mathrm{K} / \mathrm{Mg}$ com a elevação das doses de K (VELOSO et al., 2001; SPIRONELLO et al., 2004), concordando, assim, com os resultados obtidos neste trabalho. Estes resultados devem-se a elevação dos teores de $\mathrm{K}$ e aos baixos teores foliares de $\mathrm{Mg}$ (Tabelas 2 e 3). Em geral, a aplicação de doses elevadas de $\mathrm{K}$ reduz os teores foliares de $\mathrm{Ca}$ e de $\mathrm{Mg}$, devido ao antagonismo entre esses nutrientes, em função da competição pelos sítios de absorção na membrana plasmática (MEURER, 2007); no abacaxizeiro, entretanto, o aumento dos teores de $\mathrm{K}$ exerce efeito mais pronunciado na redução da absorção de Mg (SOUZA, 2000).

Os valores de referência para a relação $\mathrm{K} /$ Mg são de 5:1 para plantas com teores adequados e de 13:1 para plantas deficientes em Mg (SOUZA, 2000), confirmando, assim, os baixos teores de $\mathrm{Mg}$ apresentados na Tabela 2. Para a relação K/Ca (Tabela 3), os valores também se encontravam acima dos considerados adequados (3:1) (SOUZA, 2000).

Com base nestas informações, pode-se inferir que a não realização de calagem pode ter contribuído para maior absorção de $\mathrm{K}$ em relação ao $\mathrm{Mg}$ e aumentado os valores da relação $\mathrm{K} / \mathrm{Mg}$ (Tabela 3). Os altos valores das relações $\mathrm{K} / \mathrm{Ca}$ e $\mathrm{K} / \mathrm{Mg}$ encontrados neste trabalho são devidos aos baixos teores de $\mathrm{Ca}$ e $\mathrm{Mg}$ no solo, inferiores aos níveis críticos de implantação (MALÉZIEUX; BARTHOLOMEW, 2003) e da ausência de calagem, contribuindo para aumentar a participação do K no complexo sortivo, diminuindo, assim, os sítios de troca ocupados por $\mathrm{Ca}$ e Mg no solo (MEURER, 2007).

\section{Produção}

Os tratamentos influenciaram significativamente o peso médio dos frutos, a produtividade e o percentual de frutos das classes I e II $(p<0,05)$, sem, entretanto, influenciar sobre o percentual de frutos da classe III (Tabela 4). Os resultados concordam com os obtidos por diversos autores (OWUSU-BENNOAH, 1997; VELOSO et al., 2001; SPIRONELLO et al., 2004; SILVA et al., 2009) e corroboram as afirmações de Sousa (2000) de que o abacaxizeiro é bastante responsivo à adubação com $\mathrm{N}$ e $\mathrm{K}$, especialmente em solos com baixa capacidade de suprimento destes nutrientes.
$\mathrm{O}$ fornecimento de doses de $\mathrm{K}$ e $\mathrm{N}$ na relação $2: 1$, na maior dose de $\mathrm{N}$, com a substituição da fonte cloreto por sulfato de $\mathrm{K}$, resultou em maiores valores de peso médio de frutos $(1,38 \mathrm{~kg})$, produtividade $\left(40,6 \mathrm{t} \mathrm{ha}^{-1}\right)$ e percentual de frutos das classes II $(64,5 \%)$ e III (19,9\%), além de menor percentual de frutos da classe I (15,6\%); contudo, os valores não diferiram dos obtidos com a aplicação das doses via solo e foliar, do parcelamento das doses em cinco aplicações e da relação 1:1 na maior dose de N (Tabela 4). O melhor desempenho destes tratamentos, em termos de produtividade, coincide com maiores valores de massa fresca da folha ' $\mathrm{D}$ ', maiores teores de N e K (exceção da relação 1:1) e menores valores da relação K/N (Tabelas 2 e 3 ).

A superioridade dos dados com a utilização de sulfato de K concorda com Teixeira et al. (2011 ab). Segundo os autores, o melhor desempenho do sulfato em relação ao cloreto de $\mathrm{K}$, principalmente nas maiores doses, deve-se ao possível efeito negativo do íon cloreto (MARCHAL et al., 1981) ocorrido durante o crescimento vegetativo. Esses autores não observaram relação entre as fontes de $\mathrm{K}$ (sulfato ou cloreto) e o aumento do peso médio do fruto e, por conseguinte, dos demais componentes de produção, afirmando ser mais importante assegurar a relação $\mathrm{K}: \mathrm{N}$, que deve ser $\leq 1: 1,1$, uma vez que o efeito depressivo do íon cloreto pode ser compensado por um aumento na dose de $\mathrm{N}$.

Em relação ao parcelamento das doses em cinco aplicações, supõe-se que a mesma teria favorecido o melhor aproveitamento pelas plantas, devido à maior eficiência de absorção e redução das perdas por lixiviação, principalmente de $\mathrm{K}$, fato bastante comum em solos de textura arenosa, com baixos teores de matéria orgânica e baixa CTC (MEURER, 2007).

Quanto à aplicação foliar, os resultados devem-se a alta capacidade de absorção foliar de nutrientes pelo abacaxizeiro, em função de sua arquitetura, morfologia e anatomia (CHOAIRY et al., 1990). Por outro lado, o desempenho da relação $1: 1$ na maior dose de $\mathrm{N}$ aproxima-se da relação de 1:1,1 proposta por Marchal et al. (1981) e coincide com Silva et al. (2009) ao estimar máxima produção com $312 \mathrm{~kg} \mathrm{ha}^{-1}$ de N e $341 \mathrm{~kg} \mathrm{ha}^{-1}$ de K.

Com base no exposto, pode-se destacar a adequação das quatro estratégias de adubação para o sistema de produção de abacaxi na região de Tabuleiros Costeiros da Paraíba; contudo, a escolha por uma delas deve basear-se no destino da produção (SOUZA, 2000), bem como nos efeitos sobre a qualidade dos frutos e a economicidade das mesmas. 
TABELA 1- Caracterização dos tratamentos avaliados.

\begin{tabular}{|c|c|c|c|c|}
\hline Tratamento & Codificação $^{11}$ & Relação K/N & $\mathrm{N}$ & $\mathrm{K}$ \\
\hline & & & \multicolumn{2}{|c|}{--------------------------'g planta'-1------------------- } \\
\hline 1 & $\mathrm{~K}_{1} \mathrm{~N}_{1}$ & $0,85: 1$ & $7,2(300)$ & $6,12(255)$ \\
\hline 2 & $\mathrm{~K}_{2} \mathrm{~N}_{1}$ & $1: 1$ & $7,2(300)$ & $7,20(300)$ \\
\hline 3 & $\mathrm{~K}_{3} \mathrm{~N}_{1}$ & $2: 1$ & $7,2(300)$ & $14,4(600)$ \\
\hline 4 & $\mathrm{~K}_{4} \mathrm{~N}_{1}$ & $3: 1$ & $7,2(300)$ & $21,6(900)$ \\
\hline 5 & $\mathrm{~K}_{1} \mathrm{~N}_{2}$ & $0,85: 1$ & $10,8(450)$ & $9,18(382)$ \\
\hline 6 & $\mathrm{~K}_{2} \mathrm{~N}_{2}$ & $1: 1$ & $10,8(450)$ & $10,8(450)$ \\
\hline 7 & $\mathrm{~K}_{3} \mathrm{~N}_{2}$ & $2: 1$ & $10,8(450)$ & $21,6(900)$ \\
\hline 8 & $\mathrm{~K}_{4} \mathrm{~N}_{2}$ & $3: 1$ & $10,8(450)$ & $32,4(1350)$ \\
\hline 9 & Fontes & $2: 1$ & $10,8(450)$ & $21,6(900)$ \\
\hline 10 & Parcelamento & $2: 1$ & $10,8(450)$ & $21,6(900)$ \\
\hline 11 & Época & $2: 1$ & $10,8(450)$ & $21,6(900)$ \\
\hline 12 & Forma & $2: 1$ & $10,8(450)$ & $21,6(900)$ \\
\hline 13 & Testemunha & sem adubação & - & - \\
\hline
\end{tabular}

${ }^{1 / A s}$ plantas de todos os tratamentos, exceto a testemunha, receberam $5 \mathrm{~g}$ de superfosfato triplo aos 60 dap $\left(88 \mathrm{~kg} \mathrm{ha}^{-1} \mathrm{de}_{2} \mathrm{O}_{5}\right)$. No tratamento nove, a fonte de $\mathrm{K}$ utilizada foi sulfato de potássio, as doses foram aplicadas via solo e parceladas em três aplicações (60; 180 e 270 dap). No tratamento 10, as doses de $\mathrm{N}$ e K foram aplicadas via solo e parceladas em cinco aplicações $(60 ; 180 ; 210 ; 240$ e 270 dap). No tratamento 11, as doses de N e K foram parceladas em três aplicações (60;180 e 360 dap). No tratamento 12, as doses de N e K foram parceladas em três aplicações (60;180 e 270 dap) e aplicadas $50 \%$ no solo e $50 \%$ via foliar. Valores entre parênteses indicam as doses de $\mathrm{N}$ e $\mathrm{K}$, em kg ha-1 , considerando uma densidade de 41.666 plantas ha $^{-1}$

TABELA 2 - Valores de massa fresca da folha 'D' (MFFD) e de teores foliares de N, P, K, Ca e Mg de abacaxizeiro 'Pérola', na época de indução floral, em função das relações $\mathrm{K} / \mathrm{N}$ na adubação.

\begin{tabular}{cccccccc}
\hline Tratamento & Rel. K/N & MFFD & $\mathrm{N}$ & $\mathrm{P}$ & $\mathrm{K}$ & $\mathrm{Ca}$ & $\mathrm{Mg}$ \\
\hline $1\left(\mathrm{~K}_{1} \mathrm{~N}_{\mathrm{I}}\right)$ & $0,85: 1$ & $63,9 \mathrm{~b}$ & $12,2 \mathrm{a}$ & $1,13 \mathrm{a}$ & $20,7 \mathrm{~b}$ & $1,41 \mathrm{a}$ & $2,46 \mathrm{a}$ \\
$2\left(\mathrm{~K}_{2} \mathrm{~N}_{\mathrm{I}}\right)$ & $1: 1$ & $65,5 \mathrm{~b}$ & $11,4 \mathrm{a}$ & $1,08 \mathrm{a}$ & $20,1 \mathrm{~b}$ & $1,21 \mathrm{a}$ & $1,87 \mathrm{a}$ \\
$3\left(\mathrm{~K}_{3} \mathrm{~N}_{\mathrm{I}}\right)$ & $2: 1$ & $71,4 \mathrm{a}$ & $11,0 \mathrm{~b}$ & $1,05 \mathrm{a}$ & $23,9 \mathrm{a}$ & $1,28 \mathrm{a}$ & $2,06 \mathrm{a}$ \\
$4\left(\mathrm{~K}_{4} \mathrm{~N}_{\mathrm{I}}\right)$ & $3: 1$ & $82,8 \mathrm{a}$ & $11,7 \mathrm{a}$ & $1,13 \mathrm{a}$ & $26,2 \mathrm{a}$ & $1,20 \mathrm{a}$ & $2,05 \mathrm{a}$ \\
$5\left(\mathrm{~K}_{1} \mathrm{~N}_{2}\right)$ & $0,85: 1$ & $65,1 \mathrm{~b}$ & $11,5 \mathrm{a}$ & $1,28 \mathrm{a}$ & $21,5 \mathrm{~b}$ & $1,42 \mathrm{a}$ & $2,25 \mathrm{a}$ \\
$6\left(\mathrm{~K}_{2} \mathrm{~N}_{2}\right)$ & $1: 1$ & $75,6 \mathrm{a}$ & $10,7 \mathrm{a}$ & $1,21 \mathrm{a}$ & $21,9 \mathrm{~b}$ & $1,43 \mathrm{a}$ & $2,06 \mathrm{a}$ \\
$7\left(\mathrm{~K}_{3} \mathrm{~N}_{2}\right)$ & $2: 1$ & $67,2 \mathrm{~b}$ & $10,5 \mathrm{~b}$ & $0,90 \mathrm{a}$ & $24,8 \mathrm{a}$ & $1,87 \mathrm{a}$ & $2,18 \mathrm{a}$ \\
$8\left(\mathrm{~K}_{4} \mathrm{~N}_{2}\right)$ & $3: 1$ & $73,1 \mathrm{a}$ & $10,6 \mathrm{~b}$ & $0,99 \mathrm{a}$ & $27,8 \mathrm{a}$ & $1,67 \mathrm{a}$ & $2,13 \mathrm{a}$ \\
$9($ Fonte $)$ & $2: 1$ & $78,3 \mathrm{a}$ & $11,8 \mathrm{a}$ & $1,02 \mathrm{a}$ & $23,1 \mathrm{a}$ & $1,29 \mathrm{a}$ & $1,96 \mathrm{a}$ \\
$10($ Parcel.$)$ & $2: 1$ & $71,1 \mathrm{a}$ & $11,5 \mathrm{a}$ & $1,03 \mathrm{a}$ & $24,3 \mathrm{a}$ & $1,36 \mathrm{a}$ & $1,92 \mathrm{a}$ \\
$11($ Época $)$ & $2: 1$ & $74,7 \mathrm{a}$ & $9,6 \mathrm{~b}$ & $1,17 \mathrm{a}$ & $25,5 \mathrm{a}$ & $1,33 \mathrm{a}$ & $1,98 \mathrm{a}$ \\
$12($ Forma $)$ & $2: 1$ & $80,3 \mathrm{a}$ & $13,4 \mathrm{a}$ & $1,14 \mathrm{a}$ & $26,0 \mathrm{a}$ & $1,26 \mathrm{a}$ & $1,95 \mathrm{a}$ \\
$13($ Test. $)$ & - & $55,8 \mathrm{~b}$ & $9,3 \mathrm{~b}$ & $1,25 \mathrm{a}$ & $18,1 \mathrm{c}$ & $1,30 \mathrm{a}$ & $2,37 \mathrm{a}$ \\
Média Geral & - & 71,1 & 11,2 & 1,11 & 23,4 & 1,38 & 2,09 \\
\hline CV (\%) & - & 8,6 & 8,9 & 12,6 & 11,4 & 30,4 & 14,2 \\
\hline
\end{tabular}

Médias seguidas de uma mesma letra na coluna não diferem entre si, pelo teste de Scott-Knott, ao nível de $5 \%$ de probabilidade. 
TABELA 3 - Valores das relações K/N, K/Ca e K/Mg em tecidos foliares da folha 'D’ de abacaxizeiro 'Pérola', na época de indução floral, em função das relações K/N na adubação.

\begin{tabular}{ccccc}
\hline Tratamento & Rel. K/N & $\mathrm{K} / \mathrm{N}$ & $\mathrm{K} / \mathrm{Ca}$ & $\mathrm{K} / \mathrm{Mg}$ \\
\hline $1\left(\mathrm{~K}_{1} \mathrm{~N}_{\mathrm{I}}\right)$ & $0,85: 1$ & $1,70 \mathrm{~b}$ & $14,9 \mathrm{a}$ & $8,42 \mathrm{~b}$ \\
$2\left(\mathrm{~K}_{2} \mathrm{~N}_{\mathrm{I}}\right)$ & $1: 1$ & $1,76 \mathrm{~b}$ & $17,3 \mathrm{a}$ & $10,83 \mathrm{~b}$ \\
$3\left(\mathrm{~K}_{3} \mathrm{~N}_{\mathrm{I}}\right)$ & $2: 1$ & $2,19 \mathrm{~b}$ & $19,2 \mathrm{a}$ & $11,86 \mathrm{a}$ \\
$4\left(\mathrm{~K}_{4} \mathrm{~N}_{\mathrm{I}}\right)$ & $3: 1$ & $2,22 \mathrm{~b}$ & $22,1 \mathrm{a}$ & $12,85 \mathrm{a}$ \\
$5\left(\mathrm{~K}_{1} \mathrm{~N}_{2}\right)$ & $0,85: 1$ & $1,88 \mathrm{~b}$ & $16,3 \mathrm{a}$ & $9,75 \mathrm{~b}$ \\
$6\left(\mathrm{~K}_{2} \mathrm{~N}_{2}\right)$ & $1: 1$ & $2,04 \mathrm{~b}$ & $17,9 \mathrm{a}$ & $10,63 \mathrm{~b}$ \\
$7\left(\mathrm{~K}_{3} \mathrm{~N}_{2}\right)$ & $2: 1$ & $2,37 \mathrm{a}$ & $14,7 \mathrm{a}$ & $11,38 \mathrm{a}$ \\
$8\left(\mathrm{~K}_{4} \mathrm{~N}_{2}\right)$ & $3: 1$ & $2,62 \mathrm{a}$ & $18,6 \mathrm{a}$ & $13,30 \mathrm{a}$ \\
$9($ Fonte $)$ & $2: 1$ & $1,96 \mathrm{~b}$ & $19,3 \mathrm{a}$ & $11,75 \mathrm{a}$ \\
$10($ Parcel. $)$ & $2: 1$ & $2,13 \mathrm{~b}$ & $18,1 \mathrm{a}$ & $13,10 \mathrm{a}$ \\
$11($ Época $)$ & $2: 1$ & $2,66 \mathrm{a}$ & $20,0 \mathrm{a}$ & $12,89 \mathrm{a}$ \\
$12($ Forma $)$ & $2: 1$ & $1,94 \mathrm{~b}$ & $22,2 \mathrm{a}$ & $13,56 \mathrm{a}$ \\
$13($ Test. $)$ & - & $1,96 \mathrm{~b}$ & $14,8 \mathrm{a}$ & $7,62 \mathrm{~b}$ \\
Média Geral & - & 2,11 & 18,1 & 11,4 \\
\hline CV $(\%)$ & - & 10,8 & 29,9 & 15,7 \\
\hline
\end{tabular}

Médias seguidas de uma mesma letra na coluna não diferem entre si, pelo teste de Scott-Knott, ao nível de 5 \% de probabilidade

TABELA 4 - Valores de peso médio dos frutos (PMF), produtividade (Prod) e percentual de frutos das classes comerciais I (Classe I), II (Classe II) e III (Classe III) de abacaxizeiro 'Pérola', em função das relações K/N na adubação.

\begin{tabular}{|c|c|c|c|c|c|c|}
\hline Tratamento & Relação K/N & PMF & Prod. & Classe I & Classe II & Classe III \\
\hline & & -----kg---- & --t ha-1-- & -----------. & ------- $\%$ & 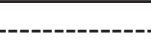 \\
\hline $1\left(\mathrm{~K}_{1} \mathrm{~N}_{1}\right)$ & $0,85: 1$ & $1,12 \mathrm{~b}$ & $33,2 \mathrm{~b}$ & $62,3 \mathrm{a}$ & $37,8 \mathrm{~b}$ & $0,0 \mathrm{a}$ \\
\hline $2\left(\mathrm{~K}_{2} \mathrm{~N}_{1}\right)$ & $1: 1$ & $1,17 \mathrm{~b}$ & $34,1 \mathrm{~b}$ & $62,2 \mathrm{a}$ & $35,6 \mathrm{~b}$ & $2,20 \mathrm{a}$ \\
\hline $3\left(\mathrm{~K}_{3} \mathrm{~N}_{1}\right)$ & $2: 1$ & $1,18 \mathrm{~b}$ & $34,9 \mathrm{~b}$ & $62,2 \mathrm{a}$ & $37,8 \mathrm{~b}$ & $0,0 \mathrm{a}$ \\
\hline $4\left(\mathrm{~K}_{4} \mathrm{~N}_{1}\right)$ & $3: 1$ & $1,18 \mathrm{~b}$ & $34,3 \mathrm{~b}$ & $62,2 \mathrm{a}$ & $33,3 \mathrm{~b}$ & $4,43 \mathrm{a}$ \\
\hline $5\left(\mathrm{~K}_{1} \mathrm{~N}_{2}\right)$ & $0,85: 1$ & $1,23 \mathrm{~b}$ & $36,6 \mathrm{a}$ & $46,7 \mathrm{a}$ & $42,2 \mathrm{~b}$ & $11,1 \mathrm{a}$ \\
\hline $6\left(\mathrm{~K}_{2} \mathrm{~N}_{2}\right)$ & $1: 1$ & $1,26 \mathrm{a}$ & $37,1 \mathrm{a}$ & $40,0 \mathrm{~b}$ & $51,1 \mathrm{a}$ & $8,9 \mathrm{a}$ \\
\hline $7\left(\mathrm{~K}_{3} \mathrm{~N}_{2}\right)$ & $2: 1$ & $1,19 \mathrm{~b}$ & $34,9 \mathrm{~b}$ & $53,3 \mathrm{a}$ & $46,7 \mathrm{~b}$ & $0,0 \mathrm{a}$ \\
\hline $8\left(\mathrm{~K}_{4} \mathrm{~N}_{2}\right)$ & $3: 1$ & $1,22 \mathrm{~b}$ & $36,4 \mathrm{a}$ & $46,7 \mathrm{a}$ & $44,4 \mathrm{~b}$ & $8,9 \mathrm{a}$ \\
\hline 9 (Fonte) & $2: 1$ & $1,38 \mathrm{a}$ & $40,6 \mathrm{a}$ & $15,6 \mathrm{~b}$ & $64,5 \mathrm{a}$ & $19,9 \mathrm{a}$ \\
\hline 10 (Parcel.) & $2: 1$ & $1,27 \mathrm{a}$ & $37,8 \mathrm{a}$ & $31,1 \mathrm{~b}$ & $62,2 \mathrm{a}$ & $6,7 \mathrm{a}$ \\
\hline 11 (Época) & $2: 1$ & $1,18 \mathrm{~b}$ & $34,9 \mathrm{~b}$ & $53,3 \mathrm{a}$ & $46,7 \mathrm{~b}$ & $0,0 \mathrm{a}$ \\
\hline 12 (Forma) & $2: 1$ & $1,34 \mathrm{a}$ & $40,2 \mathrm{a}$ & $17,8 \mathrm{~b}$ & $64,4 \mathrm{a}$ & $17,8 \mathrm{a}$ \\
\hline 13 (Test.) & - & $0,95 \mathrm{c}$ & $31,6 \mathrm{~b}$ & $84,4 \mathrm{a}$ & $15,6 \mathrm{~b}$ & $0,0 \mathrm{a}$ \\
\hline Média Geral & - & 1,20 & 36,1 & 49,1 & 44,8 & 6,15 \\
\hline CV (\%) & - & 7,50 & 7,40 & 39,8 & 32,8 & 65,0 \\
\hline
\end{tabular}

Médias seguidas de mesma letra na coluna não diferem entre si, pelo teste de Scott-Knott, ao nível de $5 \%$ de probabilidade. 


\section{CONCLUSÕES}

1- $\mathrm{O}$ aumento das relações $\mathrm{K} / \mathrm{N}$ eleva o peso da folha ' $\mathrm{D}$ ', os teores de $\mathrm{K}$ e os valores das relações $\mathrm{K} / \mathrm{N}$ e $\mathrm{K} / \mathrm{Mg}$; reduz os teores de $\mathrm{N}$ e não afeta os teores de $\mathrm{P}$, $\mathrm{Ca}$ e a relação $\mathrm{K} / \mathrm{Ca}$.

2- $\mathrm{O}$ aumento das relações $\mathrm{K} / \mathrm{N}$ não influencia no peso médio, na produtividade e no percentual de frutos das classes I, II e III.

3- A utilização de sulfato de $\mathrm{K}$, o parcelamento das doses de $\mathrm{N}$ e $\mathrm{K}$, na forma de $\mathrm{KCl}$, em cinco aplicações, e a aplicação de metade das doses de $\mathrm{N}$ e $\mathrm{K}$ via foliar na relação 2:1 aumentam o peso da folha ' $\mathrm{D}$, os teores foliares de $\mathrm{N}$ e os valores de peso médio, produtividade e percentual de frutos da classe II.

\section{REFERÊNCIAS}

BRASIL. Ministério da Agricultura. Escritório de Pesquisa e Experimentação. Equipe de Pedologia e Fertilidade do solo. I Levantamento exploratório-reconhecimento de solos do Estado da Paraíba. II Interpretação para uso agrícola dos solos do Estado da Paraíba. Rio de Janeiro: SUDENE, 1972. 638p. (Boletim Técnico, 15, Série Pedológica, 8).

CEAGESP. Programa brasileiro para modernização da horticultura: normas de classificação do abacaxi. São Paulo: Central de Qualidade em Horticultura, 2003. (Documentos, 24).

CHOAIRY, S.A.; LACERDA, J.T.; FERNANDES, P.D. Adubação líquida e sólida de nitrogênio e potássio em abacaxizeiro Smooth Cayenne na Paraíba. Pesquisa Agropecuária Brasileira, Brasília, n. 25, p. 733-737, 1990.

EMBRAPA. Centro Nacional de Pesquisa de Solos. Sistema brasileiro de classificação de solos. 2.ed. Rio de Janeiro, 2006. 212p.

EMBRAPA. Manual de métodos de análise e solos. 2.ed. rev. e atual. Rio de Janeiro: EMBRAPA/CNPS, 1997. $212 \mathrm{p}$

IBGE. Levantamento sistemático da produção agrícola. Rio de Janeiro: LSPA, 2012. Disponível em: < http://www.sidra.ibge.gov.br/cgi-bin/prtabl>. Acesso em: 15 jul. 2012.
LACOEUILHE, J.J. La fumure N-K de l'ananas em Côte d'Ivore. Fruits, Paris, v.33, n.5, p.341-348, 1978.

MALÉZIEUX, E.; BARTHOLOMEW, D.P. Plant nutrition. In: BARTHOLOMEW, D.P.; PAUL, R.E.; ROHRBACH, K.G. (Ed.). The pineapple: botany, production and uses. Honolulu: CAB, 2003. p.143165.

MARCHAL, J.; PINON, A.; TEISSON, C. Effects de la forme d'engrais potassiques sur la qualité de l'ananas en Côte d'Ívoire. Fruits, Paris, v. 36, n. 12 , p. $737-743,1981$.

MARSCHNER, H. Mineral nutrition of higher plants. $2^{\text {nd }}$ ed. San Diego: Academic Press, 1997. 889 p.

MEURER, E.J. Potássio. In: FERNANDES, M.S. (Ed.). Nutrição mineral de plantas. Viçosa: SBCS, 2007. p.281-298.

OLIVEIRA, E.F.; CARVALHO, R.A; LACERDA, J.T.; CHOAIRY, S.A.; BARREIRO NETO, M. Abacaxi: sistema de cultivo para o tabuleiro paraibano. João Pessoa: EMEPA, 2002. 38p.

OWUSU-BENNOAH, E.; AHENKORAH, Y.; NUTSUKPO. Effect of different levels of $\mathrm{N}: \mathrm{K}_{2} \mathrm{O}$ on the yield and quality of pineapple in the Forest-Savanna Ecotone of Ghana. Acta Horticulturae, Leuven, n. 425, p.393-402, 1997.

RAZZAQUE, A.H.M.; HANAFI, M.M. Effects of potassium on growth, yield and quality of pineapple in tropical peat. Fruits, Paris, n.56, p. 45-49, 2001.

REINHARDT, D.H.C.; CUNHA, G.A.P. Abacaxi produção: aspectos técnicos. Brasília: SPI, 2000. $77 \mathrm{p}$.

RODRIGUES, A.A.; MENDONÇA, R.M.N.; SILVA, A.P.; SILVA, S.M.; PEREIRA, W.E. Desenvolvimento vegetativo de abacaxizeiros 'Pérola' e 'Smooth Cayenne' no Estado da Paraíba. Revista Brasileira de Fruticultura, Jaboticabal, v. 32, n.1, p. $126-134,2010$ 
SILVA, A.P.; ALVAREZ V., V.H.; SOUZA, A.P.; NEVES, J.C.L.; NOVAIS, R.F.; DANTAS, J.P. Sistema de recomendação de fertilizantes e corretivos para a cultura do abacaxizeiro. Revista Brasileira de Ciência do Solo, Viçosa, MG, v.33, n.5, p.12691280, 2009.

SOARES, A.G.; TRUGO, L.C.; BOTREL, N.; SOUZA, L.F.S. Reduction of internal browning of pineapple fruit (Ananas comosus L.) by preharvest soil application of potassium. Postharvest Biology and Technology, Pullman, v.35, p. 201-207, 2005.

SOUZA, L.F.S. Adubação. In: REINHARDT, D.H.; SOUZA, L.F.S.; CABRAL, J.R.S. Abacaxi produção: aspectos técnicos. Brasília: EMBRAPA, 2000. p. 30-34.

SOUZA, C.B.; SILVA, B.B.; AZEVEDO, P.V. Crescimento e rendimento do abacaxizeiro nas condições climáticas dos Tabuleiros Costeiros do Estado da Paraíba. Revista Brasileira de Engenharia Agricola e Ambiental, Campina Grande, v. 11, n.2, p.134-141, 2007.

SPIRONELLO, A.; QUAGGIO, J.A.; TEIXEIRA, L.A.J.; FURLANI, P.R.; SIGRIST, J.M.M. Pineapple yield and fruit quality effected by NPK fertilization in a tropical soil. Revista Brasileira de Fruticultura, Jaboticabal, v. 26, n.1, p. 155-159, 2004.
TEDESCO, M.J.; GIANELLO, C.; BISSANI, C.A.; BOHNEN, H.; VOLKWEISS, S.J. Análise de solo, planta e outros materiais. Porto Alegre: UFRGS, 1995. $174 \mathrm{p}$.

TEIXEIRA, L.A.J.; QUAGGIO, J.A.; CANTARELLA, H.; MELLIS, E.V. Potassium fertilization for pineapple: effects on plant growth and fruit yield. Revista Brasileira de Fruticultura, Jaboticabal, v.33, n.2, p.618-626, 2011a.

TEIXEIRA, L.A.J.; QUAGGIO, J.A.; CANTARELLA, H.; MELLIS, E.V. Potassium fertilization for pineapple: effects on soil chemical properties and plant nutrition. Revista Brasileira de Fruticultura, Jaboticabal, v.33, n.2, p.627-636, 2011 b.

TEIXEIRA, L.A.J.; SPIRONELLO, A.; FURLANI, P.R.; SIGRIST, J.M.M. Parcelamento da adubação NPK em abacaxizeiro. Revista Brasileira de Fruticultura, Jaboticabal, v.24, n.1, p. 219-224, 2002.

VELOSO, C.A.C.; OEIRAS, A.H.L.; CARVALHO, E.J.M.; SOUZA, F.R.S. Resposta do abacaxizeiro à adição de nitrogênio, potássio e calcário em Latossolo Amarelo do Nordeste Paraense. Revista Brasileira de Fruticultura, Jaboticabal, v. 23, n.1, p. 396-402, 2001. 